Magna Scientia Advanced Research and Reviews

eISSN: 2582-9394

Cross Ref DOI: $10.30574 / \mathrm{msarr}$

Journal homepage: https://magnascientiapub.com/journals/msarr/

\title{
Ultrastructural changes of the placenta in cases of preeclampsia
}

\author{
Abdelghany Hassan Abdelghany 1, *, Ahmed Abdelghany Hassan 1, Sarah Abdelghany Hassan ${ }^{2}$ and Rania \\ Mohamed Fawzy 1 \\ ${ }^{1}$ Faculty of medicine, Alexandria University, Alexandria, Egypt. \\ 2 Faculty of Dentistry, Arab Academy for science, technology and maritime transport, Alamein, Egypt.
}

Magna Scientia Advanced Research and Reviews, 2021, 03(02), 047-060

Publication history: Received on 20 October 2021; revised on 23 November 2021; accepted on 25 November 2021

Article DOI: https://doi.org/10.30574/msarr.2021.3.2.0080

\begin{abstract}
The placenta plays vital roles during fetal development and growth. The ultrastructure of the placenta together with remodeling of the uterine spiral arteries are very important to maintain the utero-placental blood flow. Preeclampsia (PE) is a multifactorial disorder with abnormal placentation affecting the mother and fetus. The aim of this study was to study the ultrastructural abnormalities of the placenta in cases of PE. The placentas of 10 PE women and 10 controls were studied. Women of PE group were delivered by caesarian section while seven control women were delivered vaginally, and three by caesarian section. Placental samples were studied both morphologically and histologically by light and transmission electron microscopy. Light microscopic study of control placentas showed numerous microvilli, few syncytial knots, thin-walled blood vessels. PE placentas showed reduced number of microvilli with numerous syncytial knots, thick-walled vessels, edematous spaces, fibrotic areas and fibrinoid degeneration. Electron microscopic study of the control placentas showed a thick layer of syncytiotrophoblast (Sy), numerous microvilli and a thin layer of cytotrophoblast (Cy). PE placenta showed hypertrophy of Cy with atrophy of Sy and scarce microvilli. The trophoblast showed edematous vacuoles and glycogen storage areas. The villous core had congested capillaries, edematous spaces, glycogen storage areas and widespread areas of fibrosis.
\end{abstract}

All the changes in PE placentas were attributed to hypoxia and oxidative stress and reduced utero-placental flow due to abnormal remodeling of the uterine spiral arteries that was aggravated by the thick placental barrier and the presence of edema, fibrosis and glycogen storage areas.

Keywords: Preeclampsia; Placenta; Syncytiotrophoblast; Cytotrophoblast; Hypoxia; Oxidative stress

\section{Introduction}

The intrauterine existence of the fetus depends upon a vital organ; the Placenta. It is essential for maintenance of pregnancy and fetal growth and development [1]. During its transient existence, it performs a lot of functions that will be taken later on by different organs as the lungs, liver, gut, kidneys and endocrine glands. Its principal function is to supply the fetus with oxygen and nutrients [2,3]. The structure of the mature placenta has been dealt with in detail elsewhere; its fetal surface presents a tree of chorionic villi that invade the uterine vessels to let the maternal blood reach the intervillous spaces. The villi are covered by a multinucleated syncytial layer, the syncytiotrophoblast (Sy) and a deeper thin layer of cytotrophoblast (Cy) which gets thinner as pregnancy proceeds but persists until term [2]. The core of the chorionic villi shows the fetal capillaries where the maternal and fetal bloods are separated by the placental barrier which is formed of Sy, Cy, endothelial lining of the fetal capillaries and connective tissue core of the villi [4]. During pregnancy, the uterine spiral arteries undergo remodeling; loss of smooth muscle cells from their walls; resulting in their dilatation that will help reduction of the velocity of the maternal blood flow [5].

\footnotetext{
${ }^{*}$ Corresponding author Abdelghany Hassan Abdelghany

Faculty of medicine, Alexandria University, Alexandria, Egypt.
} 
Hypertensive disorders are common pregnancy complications leading to deleterious outcomes for the mother and fetus. Preeclampsia (PE) is one of those most serious disorders affecting 5-7\% of all pregnancies and causes about 70,000 maternal and 500,000 fetal deaths every year worldwide [6,7].

$\mathrm{PE}$ is a multifactorial disease that develops after 20 weeks of gestation and although the underlying pathophysiology is not clear, abnormal placentation has been suggested. The shallow trophoblast migration towards the uterine spiral arterioles and the impaired remodeling of these arteries leads to a hypoperfused placenta, thus creating a favorable environment for developing hypoxia and oxidative stress (OS) $[8,9,10,11]$.

Ischemic placenta releases factors causing maternal vascular endothelial dysfunction, systemic vasoconstriction, OS, and intrauterine growth restriction with neonatal low birth weight. Currently, treatment of PE is limited to treatment of hypertension and in severe cases, it may require premature labor induction with its comorbidities as respiratory distress syndrome $[11,12]$. It may be complicated by devastating consequences as stroke, kidney failure and eclampsia $[9,11]$.

As the placenta is the central organ in PE, this study was conducted to detect the ultrastructural changes of the placenta in pregnancies complicated by PE.

\section{Material and methods}

The placentas of 20 women, 10 control and 10 with PE, were studied with free consents approved by the Committee of Ethics in the Faculty of Medicine, Alexandria University. Women with a previous history of diabetes, hypertension or other chronic diseases were excluded. The age of the women ranged from 23 to 29 years for the controls and 25 to 32 years for PE group. The diagnosis of PE was made based on the criteria of the WHO as new-onset systolic blood pressure $>140 \mathrm{mmHg}$ and diastolic blood pressure $>90 \mathrm{mmHg}$ plus new-onset proteinuria $(>300 \mathrm{mg} / 24 \mathrm{~h}$ ) $[13,14]$.

All women of the PE group were delivered by caesarian section at 34 to 35 weeks of gestation. Seven control women were delivered vaginally, while three were delivered by caesarian section at 36 to 39 weeks. All deliveries were done at the Department of Gynecology and Obstetrics, Faculty of Medicine, Alexandria University. The neonatal birth weight $(\mathrm{kg})$ and the placental weight $(\mathrm{gm})$ together with the placental diameter $(\mathrm{cm})$ were measured. Sections $(0.5 \mu \mathrm{m})$ of whole thickness placental tissues were prepared and stained with hematoxyline and eosin (HE) and Masson trichrome stain to detect the presence of fibrosis; and the sections were examined under Optika B-150 (Optika SRL, Ponteranica, Italy) light microscope. The sections were analyzed by Imag J software to quantify the amount of fibrosis [15]. Blocks for electron microscopy were fixed in the fixative $3 \%$ glutaraldehyde and $2 \%$ paraformaldehyde in $0.1 \mathrm{~mol} / \mathrm{l}$ cacodylate buffer ( $\mathrm{pH} 7.3$ ) for $24 \mathrm{~h}$ at $4^{\circ} \mathrm{C}$. After fixation in $1.0 \% 0$ sO4 in $0.1 \mathrm{~mol} / \mathrm{l}$ cacodylate buffer (pH 7.3) for $2 \mathrm{~h}$ at room temperature, the tissue specimens were subjected to dehydration in graded ethanol series. After immersion in propylene oxide (three times for $10 \mathrm{~min}$ each), the samples were immersed overnight in a mixture (1:1) of propylene oxide and Epon 812 resin (Sigma Aldrich, St. Louis, MO, USA) to be finally embedded in Epon-812 resin. Semithin sections $(0.5 \mu \mathrm{m}$ thick) were cut using an ultramicrotome (Leica Ultracut; Leica, Berlin, Germany) that were picked on copper grids and counterstained with $2 \%$ uranyl acetate and lead citrate [16]. The specimens were then examined by transmission electron microscopy JEM-100CXi, (Jeol, Tokyo, Japan) at the electron microscope unit of Faculty of Science, Alexandria University. The results were analyzed statistically using SPSS 22 (SPSS Inc, Chicago, IL, USA). Mean \pm standard deviation (SD) of the two groups were compared using paired-samples t-test and the level of significance was accepted as $\mathrm{p}<0.05$.

\section{Results}

\subsection{Morphometric results}

The present study recorded significant changes in cases of PE as reduction of the gestational age and neonatal weight together with reduction of morphometric data regarding the placental diameter and weight when compared with control placentas. (Table 1). 
Table 1 Parameters of the studied control and PE placentas

\begin{tabular}{|c|c|c|c|c|c|}
\hline \multicolumn{2}{|l|}{ Variables } & Control & PE & t-test & p-value \\
\hline \multirow{3}{*}{ Gestational age (weeks) } & Minimum & 36 & 34 & \multirow{3}{*}{10.83} & \multirow{3}{*}{$0.01^{*}$} \\
\hline & Maximum & 39 & 35 & & \\
\hline & Mean \pm SD & $36.53 \pm 0.44$ & $34.12 \pm 0.45$ & & \\
\hline \multirow{3}{*}{ Placental diameter $(\mathrm{cm})$} & Minimum & 17.5 & 16 & \multirow{3}{*}{7.95} & \multirow{3}{*}{$0.00^{*}$} \\
\hline & Maximum & 19 & 18 & & \\
\hline & Mean \pm SD & $18.69 \pm 0.35$ & $17.45 \pm 0.34$ & & \\
\hline \multirow{3}{*}{ Placental weight (gm) } & Minimum & 460 & 380 & \multirow{3}{*}{19.57} & \multirow{3}{*}{$0.01^{*}$} \\
\hline & Maximum & 490 & 410 & & \\
\hline & Mean \pm SD & $481 \pm 14.41$ & $399.6 \pm 10.77$ & & \\
\hline \multirow{3}{*}{ Neonatal weight (kg) } & Minimum & 2.80 & 2.40 & \multirow{3}{*}{11.20} & \multirow{3}{*}{$0.01^{*}$} \\
\hline & Maximum & 3.10 & 2.60 & & \\
\hline & Mean \pm SD & $2.97 \pm 0.13$ & $2.46 \pm 0.12$ & & \\
\hline
\end{tabular}

\subsection{Histological results}

\subsubsection{Light microscopic results}

H\&E stain

Light microscopy of the control placenta showed a large number of small sized and densely packed microvilli with narrow intervillous spaces filled with blood cells. Few syncytial knots ; which are aggregations of the nuclei of the Sy on the surface of the microvilli; were seen at the poles of the microvilli. The villous core exhibited small thin-walled blood vessels containing blood cells (Figure 1). In PE placenta, the number of microvilli was reduced with relatively more syncytial knots when compared with the control together with wider intervillous spaces. The villous core in a number of microvilli exhibited areas of fibrosis and scattered spaces of edema. Also, a number of microvilli showed dilated blood vessels with thickening of their walls. There were areas of villous and perivillous fibrinoid degeneration (Figure 2).
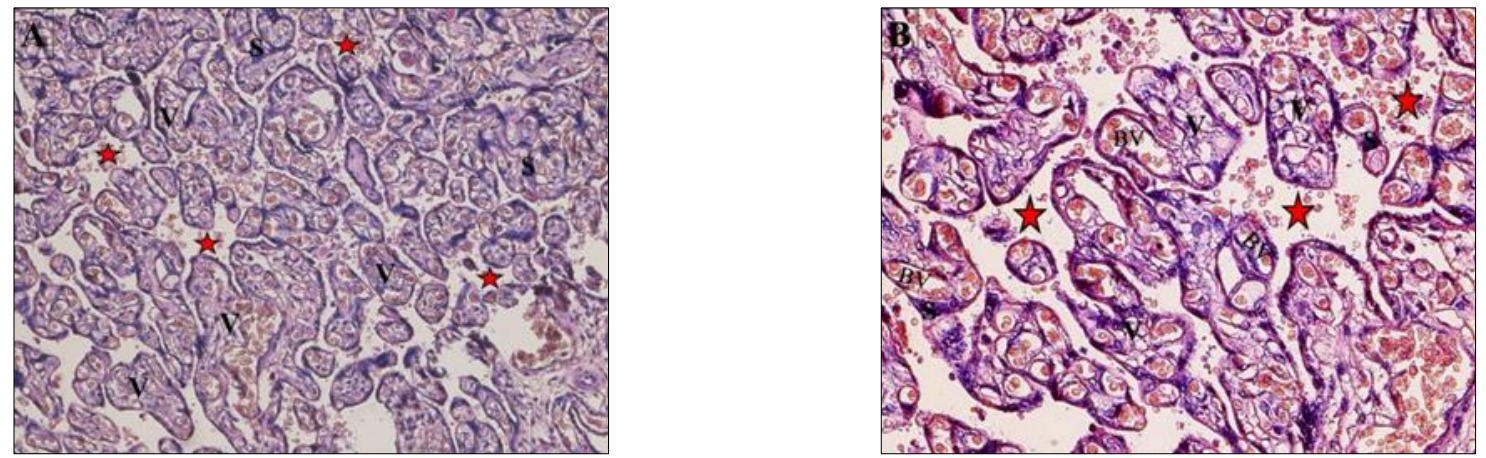

Figure $1(A, B)$ A Photomicrograph of a control placenta showing numerous small-sized microvilli (V), few syncytial knots $(\mathrm{S})$ and narrow intervillous spaces (red star) filled with blood cells. The villous core shows thinwalled blood vessels (BV) containing blood cells. Haematoxylene and Eosin stain, $\times 200$ (A); $\times 400$ (B). 

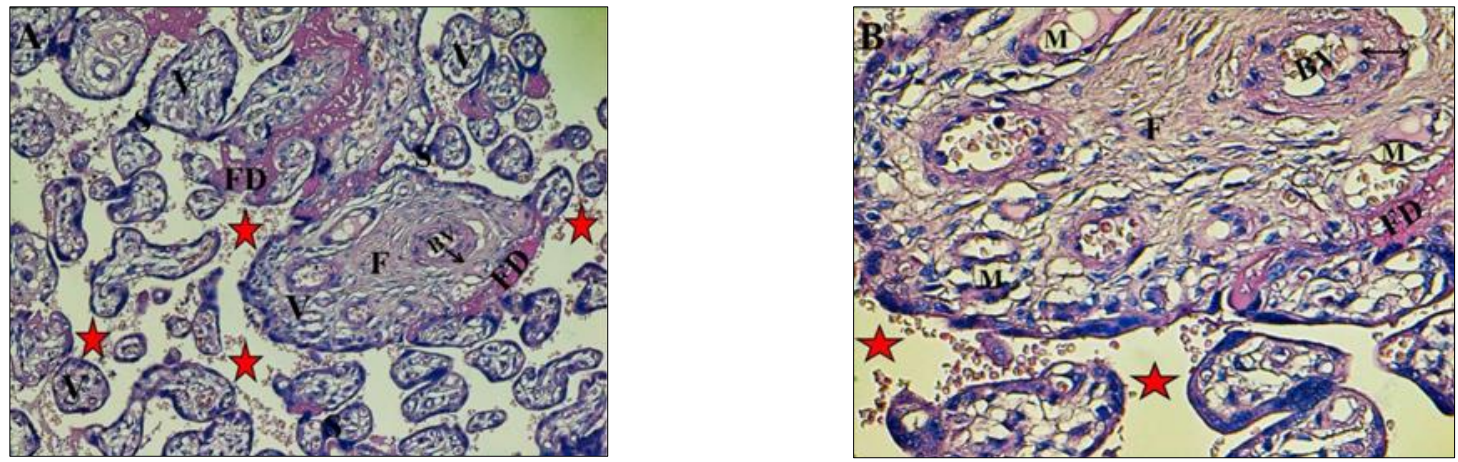

Figure 2 (A, B) A photomicrograph of PE placenta showing reduced number of microvilli (V) with numerous syncytial knots (S) and wider intervillous spaces (red star) containing blood cells. Areas of fibrosis (F), spaces of edema (M) and thick-walled (black arrow) blood vessels (BV) containing blood cells are seen in the villous

core. There are areas of villous and perivillous fibrinoid degeneration (FD) seen in a bright pink color. Haematoxylene and Eosin stain, $\times 200(A) ; \times 400(B)$.

Masson trichrome stain

Microscopic examination of the control placenta showed no or negligible fibrosis in the core and the thin-walled blood vessel walls of the placental villi. (Figure 3A) On the other hand, the terminal villi of the PE placentas showed significantly wider areas of fibrosis in the villous mesenchymal core and in the thick walls of the blood vessels (Figure 3B,C).
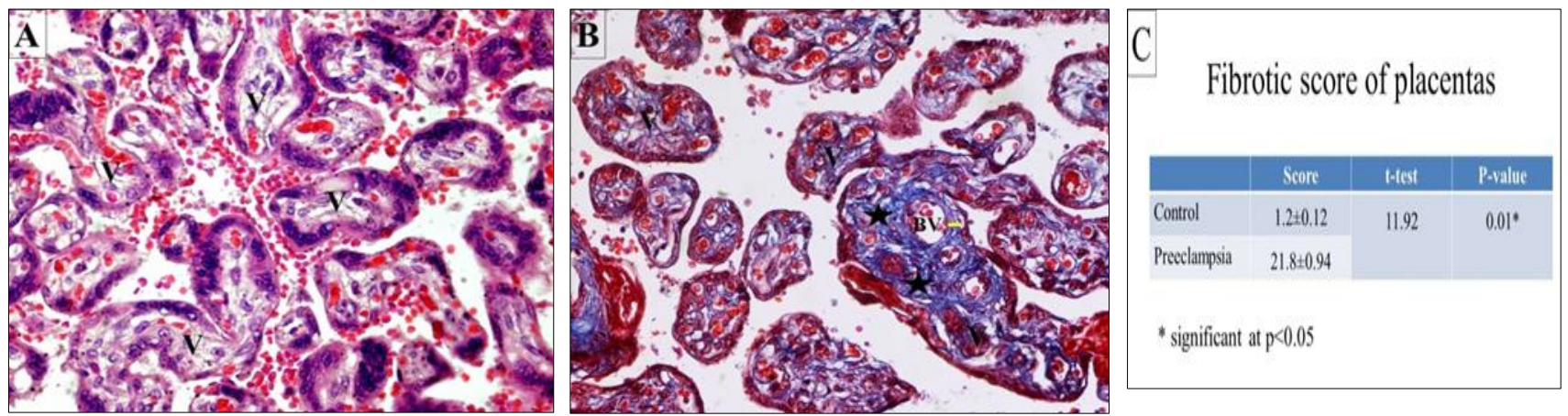

Figure 3 A photomicrograph of control (A) and PE (B) placentas. The control placenta shows negligible fibrosis in the chorionic villi (V) while the PE placenta shows wider areas of fibrosis seen as bluish coloration. The fibrotic areas are seen in the villous core (black star) and in the thick walls of the blood vessels (yellow line). The amount of fibrous tissue is significantly increased in the PE placenta (C). Masson TrichromeX400

\subsubsection{Electron microscopic results}

Electron microscopic study of the control full-term placenta showed that the terminal chorionic villi had a covering of a thick layer of Sy which had a lot of cylindrical microvilli (Figures 4, 5,6). The cytoplasm of Sy was relatively dense surrounding multiple nuclei with rough endoplasmic reticulum (rER) (Figures 4, 5,6). Deeper to SY there was a thin layer of Cy where the cells had a pale cytoplasm that harbored some rER cisternae. (Figure 5). In some areas of the villi, there was only Sy, without Cy (Figures 4,6). The underling villous core demonstrated fetal capillaries that contained red blood cells (RBCs) and were lined with flat endothelial cells (Figures 6,7). The capillaries were externally limited by concentric layers of reticular fibers (Figures 6,7).

The PE placenta showed a thin atrophic layer of Sy exhibiting scarce irregular microvilli. The underlying Cy was thick and rich of organelles (Figures 8,9,10). The cytoplasm of the Sy showed a lot of vacuoles of diverse sizes and shapes representing edematous spaces with aggregates of glycogen granules with the presence of lysosomes (Figures 10,11). The cells of the Cy showed vesicular nuclei and the cytoplasm was rich in ribosomal granules and showed a lot of mitochondria with some vesicles representing edematous spaces (Figures 9,11). The subtrophoblastic membrane was obviously thick and the mesenchymal core of the villous had telocytes, fibroblasts and multiple areas of fibrosis 


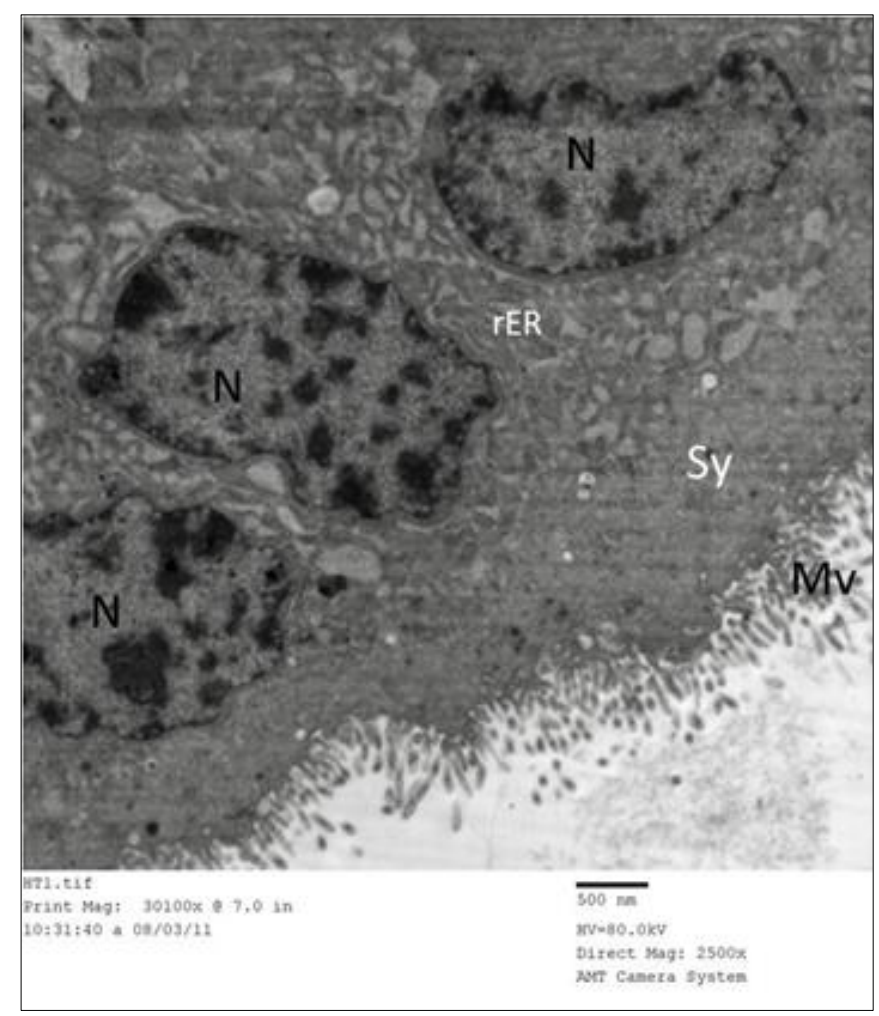

Figure 4 An electron micrograph of a terminal chorionic villus of a control placenta showing that the covering trophoblast has thick Sy with a dense cytoplasm surrounding multiple nuclei (N) and containing a lot of rER.. Multiple cylindrical microvilli (Mv) are shown on the surface. Magnification $\times 2500$, Scale bar $=500 \mathrm{~nm}$

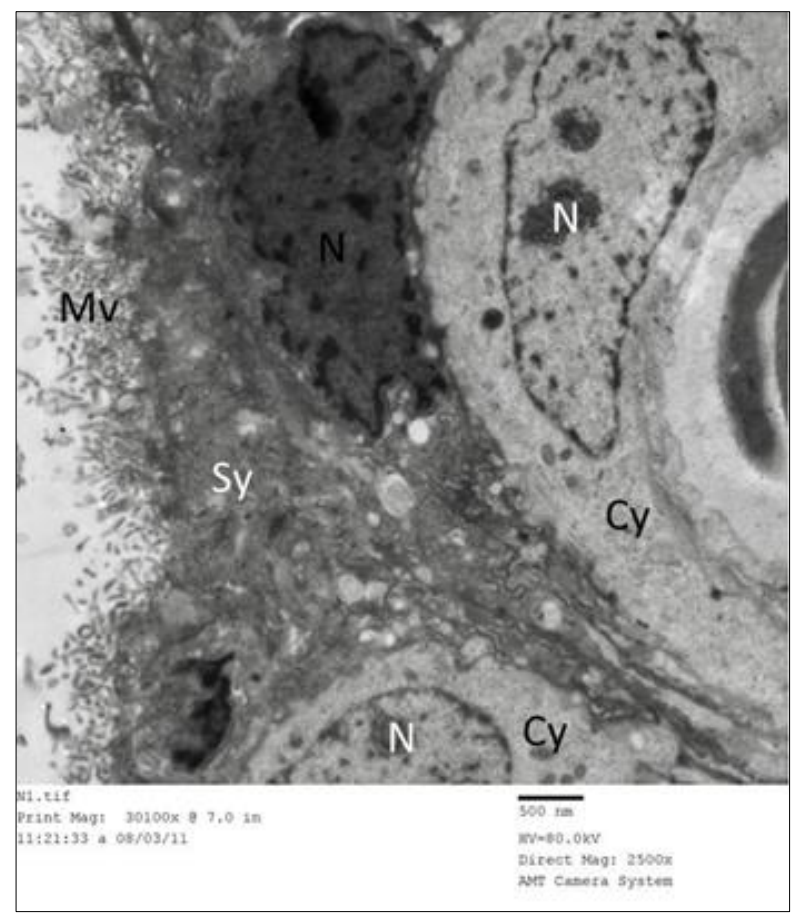

Figure 5 An electron micrograph of a terminal chorionic villus of a control placenta showing the thick covering Sy with the relatively dense cytoplasm with multiple cylindrical microvilli $(\mathrm{Mv})$ shown on the surface while the underlying Cy is thinner and shows pale cytoplasm. Nuclei $(\mathrm{N})$. Magnification $\times 2500$, Scale bar $=500 \mathrm{~nm}$ 


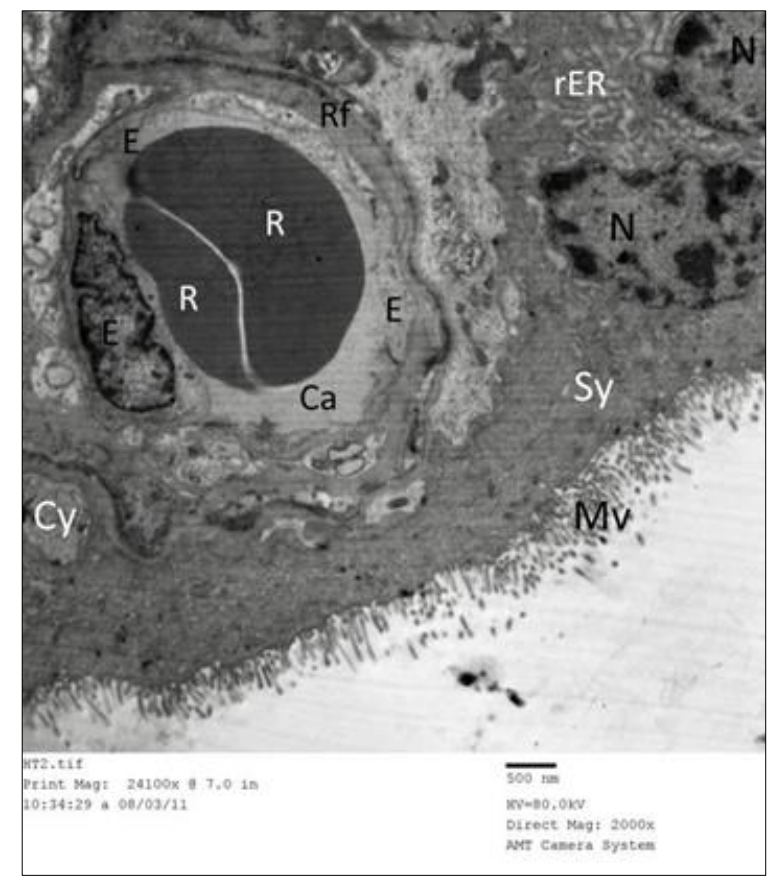

Figure 6 An electron micrograph of a terminal chorionic villus of a control placenta showing the thick Sy with its dense cytoplasm containing a lot of rER and multiple nuclei $(\mathrm{N})$ with multiple cylindrical microvilli (Mv). The underlying thin $\mathrm{Cy}$ is absent in some regions. The villous core shows a fetal capillary (Ca) which is lined by endothelial cells (E) and surrounded by reticular fibers (Rf). Cytotrophoblast (CY), RBCs (R). Magnification $\times 2000$, Scale bar $=500 \mathrm{~nm}$

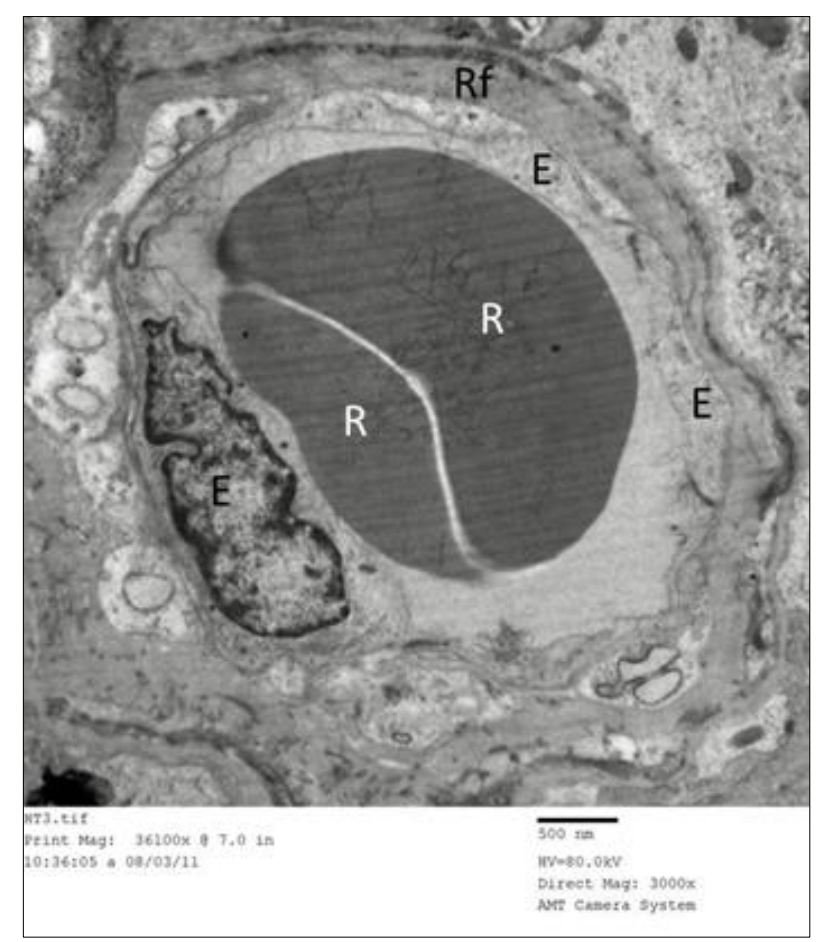

Figure 7 Detailed ultrastructure of a fetal capillary of a control placenta. This capillary is lined by flat endothelial cells (E). The capillary is externally limited by concentric arrays of reticular fibers (Rf). RBCs (R). Magnification $\times 3000$, Scale bar $=500 \mathrm{~nm}$ 


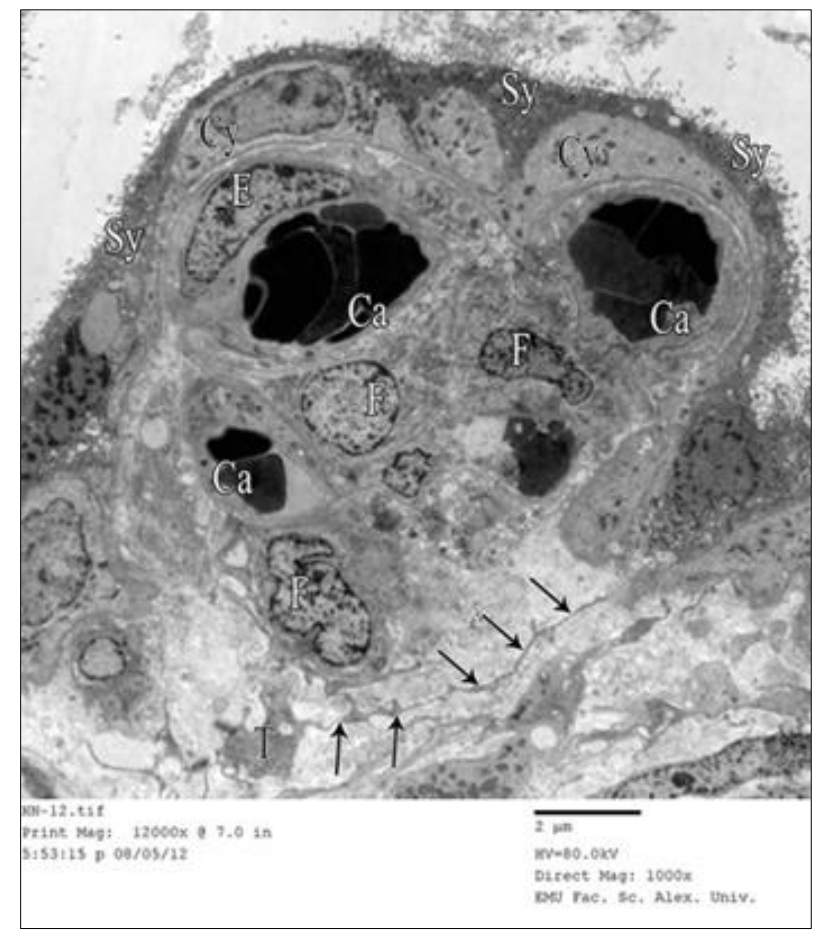

Figure 8 Electron micrograph of a terminal chorionic villus of a PE placenta. It is covered by an atrophied layer of Sy that is separated from the congested fetal capillary $(\mathrm{Ca}$ ) by a thick layer of organelles-rich Cy. The capillary endothelium (E) is bulging while the villus core exhibits fibroblasts (F) and telocytes (T) characterized by long thin irregular processes (arrows). Magnification x 1000, bar - 500nm

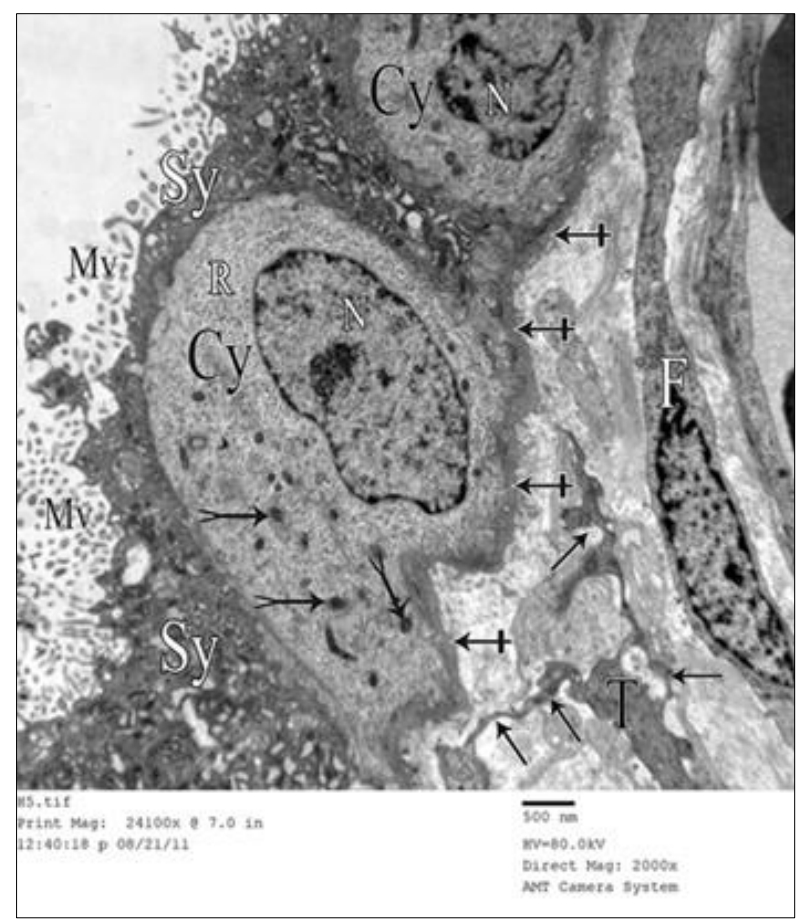

Figure 9 Electron micrograph of a terminal chorionic villus of a PE placenta. The Cy forms a thick layer that is covered by an atrophied dense cytoplasm of Sy that bears irregular scarce microvilli (Mv). The cells of Cy contain vesicular nuclei $(\mathrm{N})$ and their cytoplasm is rich in ribosomal granules (R) and mitochondria (forked arrows). The sub-trophoblastic basement membrane (crossed arrows) is obviously thick and the villus mesenchymal core reveals a fibroblast (F) and a telocyte (T) with its characteristic long irregular processes (arrows). Magnification x 2000, bar - 500nm 


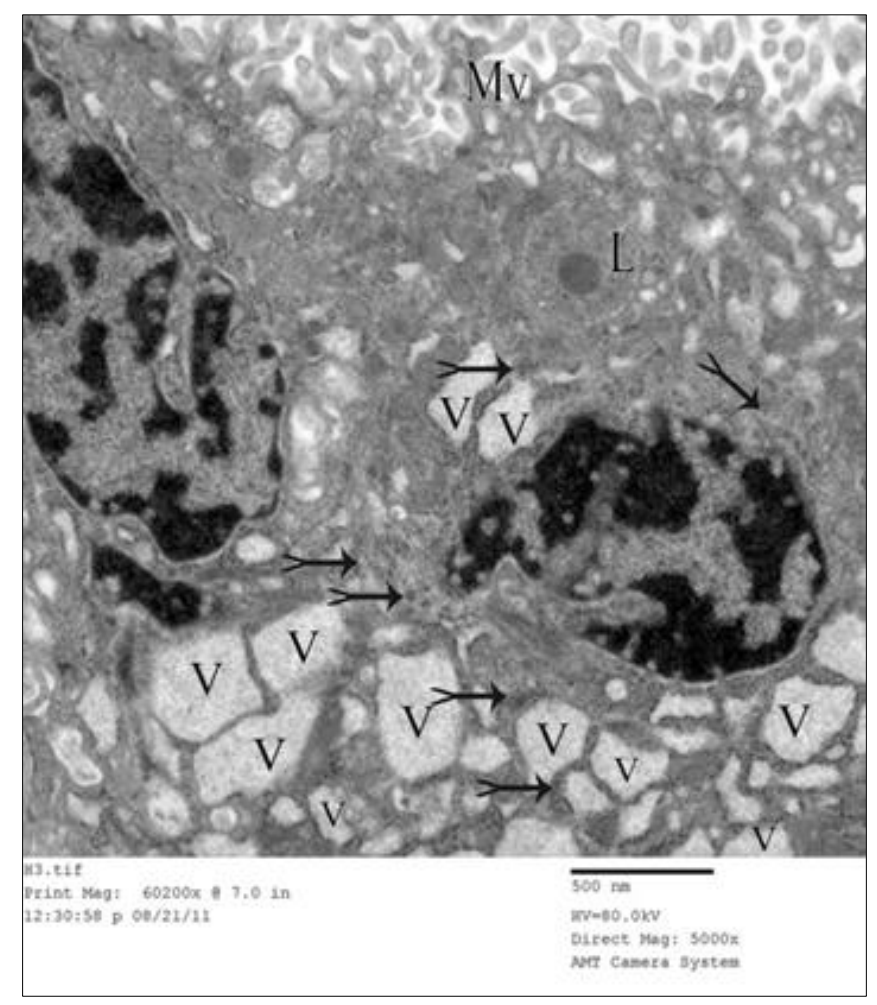

Figure 10 A magnified part of Sy of a terminal chorionic villus of a PE placenta with its surface microvilli (Mv). A lot of vacuoles $(\mathrm{V})$ of diverse sizes and shapes occupy most of the cytoplasm that shows aggregates of glycogen granules (forked arrows). A secondary lysosome (L) appears in the apical part. Magnification x 5000, bar - 500nm

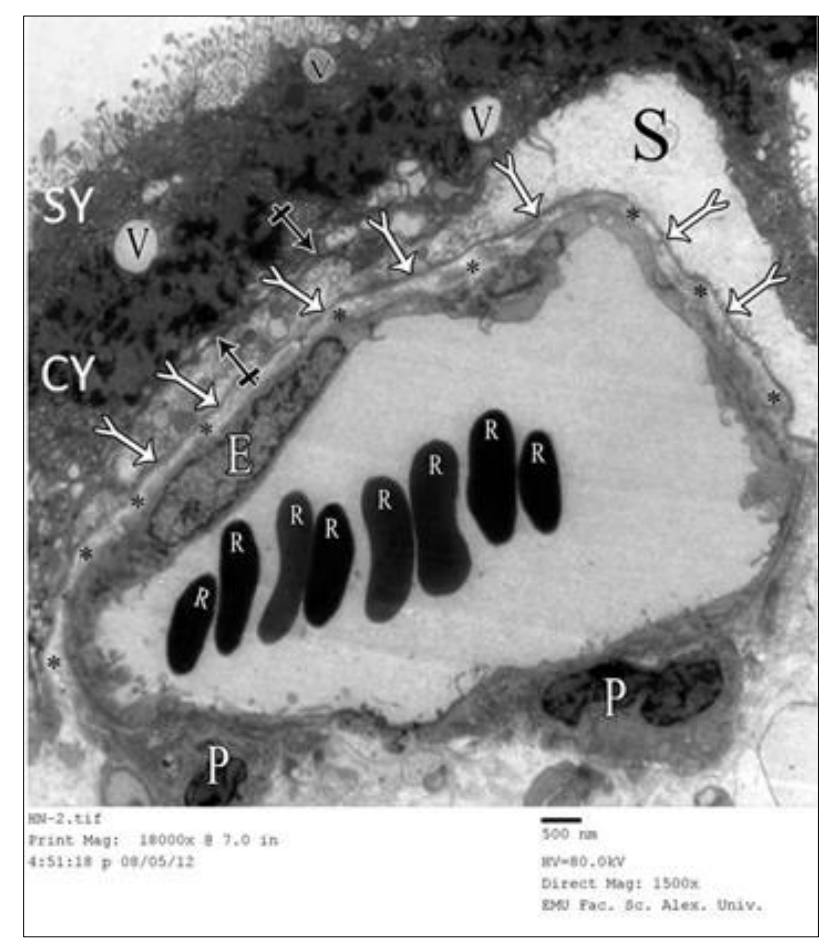

Figure 11 Electron micrograph of a PE placenta reveals atrophic Sy and thick Cy and both contain large cytoplasmic vacuoles (V). Edema forms clear spaces (S) under the trophoblast and between $\left({ }^{*}\right)$ the capillary endothelium (E) and the thickened sub-end othelial basement membrane (forked arrows). Sub- trophoblastic basement membrane (crossed arrows), pericytes (P) and fetal RBCs (R) in rouleoux arrangement. Magnification x 1500, bar - 500nm 


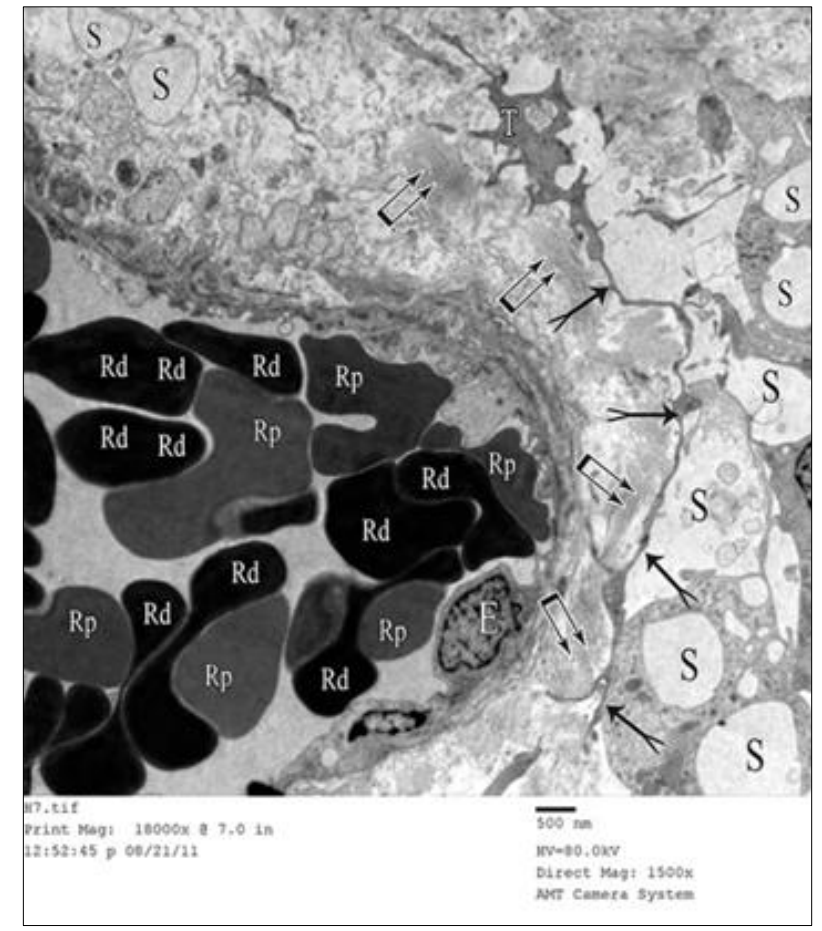

Figure 12 Electron micrograph of an extremely dilated fetal capillary of a chorionic villus of a PE placenta that is lined with bulging endothelium (E) and contains a lot of dense dark (Rd) and few pale (Rp) red blood cells. The villous core reveals multiple edematous spaces (S) and a telocyte (T) with its long thin irregular processes (forked arrows) associated with areas of fibrosis (double arrows). Magnification x 1500, bar $-500 \mathrm{~nm}$

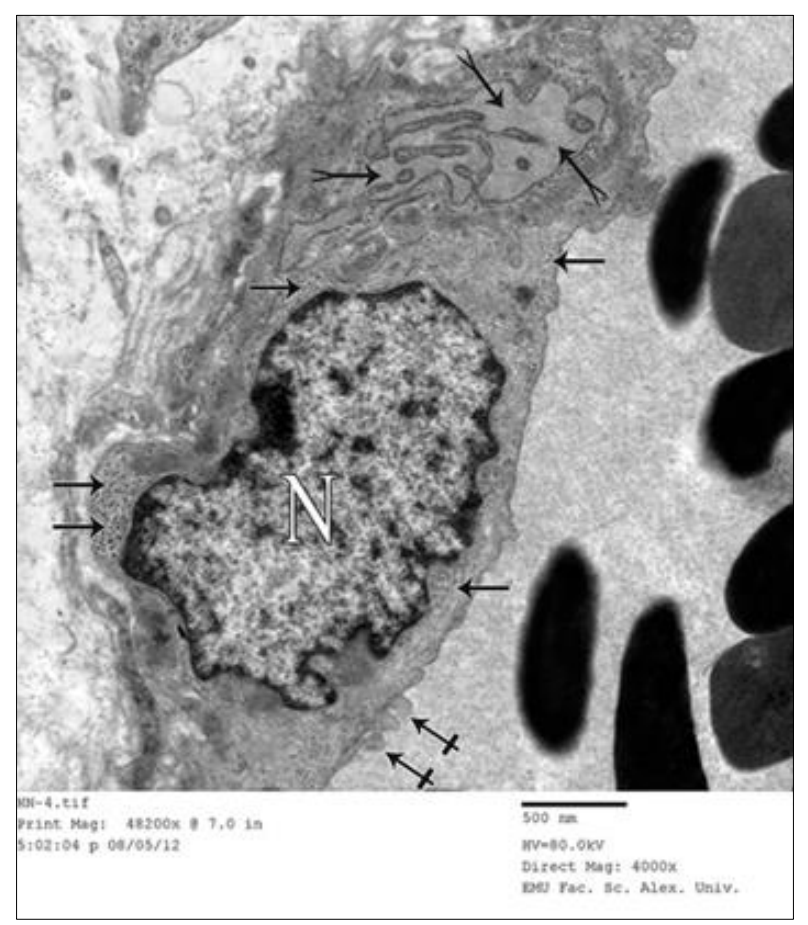

Figure 13 Electron micrograph of a high magnification of a fetal capillary of a PE placenta. One endothelial cell shows aggregates of glycogen granules (arrows) stored particularly deep to the nucleus (N), while its neighboring cell reveals dilated cisternae of endoplasmic reticulum (forked arrows) where a homogeneous material of moderate electron density is stored. Notice blebbing of capillary endothelial cytoplasm into the capillary lumen (crossed arrows). Magnification x 4000, bar - 500nm 


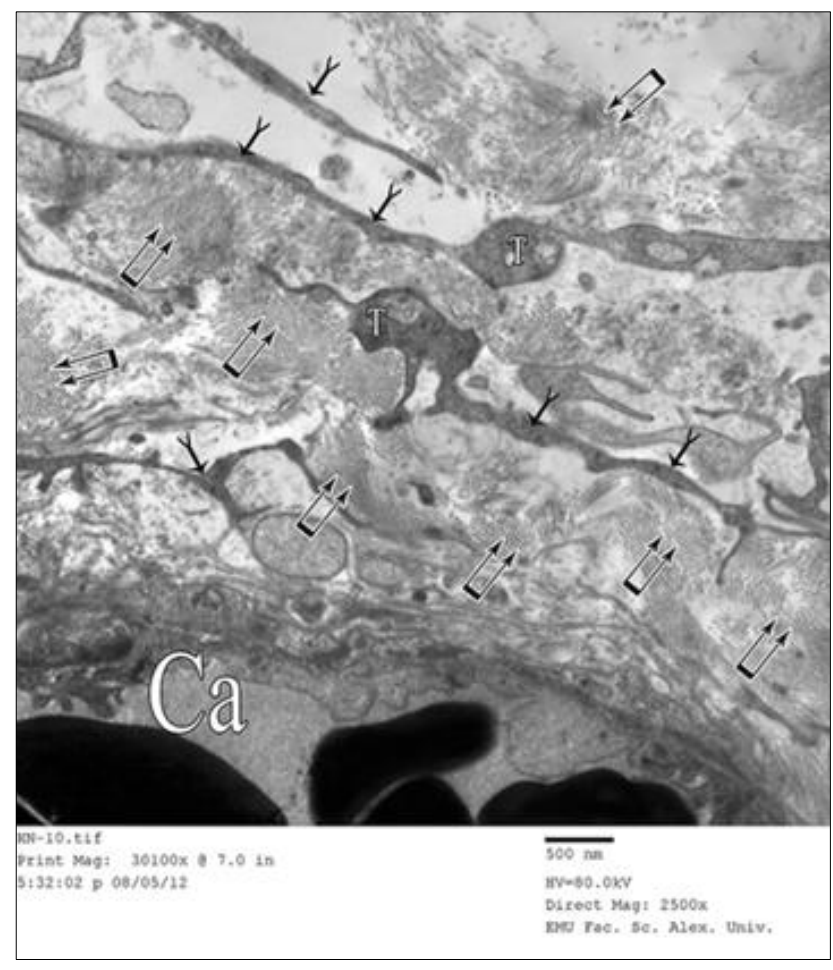

Figure 14 Electron micrograph of the core of a terminal chorionic villus of a PE placenta demonstrating the prevalence of telocytes (T) with their long processes (forked arrows) and widespread fibrosis (double arrows) around a fetal capillary (Ca) in a terminal chorionic villus. Magnification x 2500, bar - 500nm

(Figures 8,9,12). Edematous spaces were seen in the villous core and under the subtrophoblastic membrane (Figures 11,12). The villous core had congested blood vessels that contained a lot of dense dark and few pale RBCs and showed bulging endothelial cells. Some capillaries showed edematous spaces between the endothelial cells and the subend othelial membrane (Figures $8,11,12$ ). The endothelial cells showed aggregates of glycogen granules deep to the nucleus and dilated cisternae of endoplasmic reticulum where a homogeneous material of moderate electron density was stored (Figure 13). There were widespread areas of fibrosis around the villous capillaries in the villous core. (Figure 14).

\section{Discussion}

The placenta is considered the most important organ of the body for its vital roles during fetal growth, but unfortunately, it is poorly understood. The human fetus is totally dependent upon the placenta which is a mirror reflecting changes of the maternal and fetal status. Thus, pregnancy complications as PE are significantly expressed in the placenta microscopically as well as macroscopically [1,2,17].

The current study has been undertaken to show morphometric as well as structural alterations of the PE placentas. There were significantly less placental weight and diameter together with significantly less gestational age and low neonatal birth weight in cases of PE when compared with control placentas. Similar results were also mentioned in other studies $[1,14,18]$.

It has been stated that the intrauterine environment in cases of PE may alter the physiology and metabolism of the placenta and neonate. Thus, the current findings cope with the underlying pathological conditions in PE with vasculopathies of the spiral arteries and the resulting hypoxia that cause reduced utero-placental blood flow and transplacental exchange of oxygen and nutrients; hence the smaller placenta [1]. These changes together with the characteristic proteinuria of PE might interfere with the fetal nutrition and growth leading to low birth weight babies. Moreover, the less gestational age and preterm birth associated with PE might contribute to the low neonatal birth weight $[1,19,20]$. 
The control placenta in the current study showed terminal villi having a thick layer of multinucleated Sy and a thin layer of Cy with a thin BM. The terminal villi beard numerous cylindrical microvilli and the villous core showed capillaries lined with flat endothelial cells with a thin BM.

Placental growth and microvillous density are dependent upon the thickness of the trophoblastic layers that affects the feto-maternal exchange [17]. The placental barrier that separates the maternal and fetal circulations is made up of Sy, a thin layer of $\mathrm{Cy}$, a thin layer of vascular endothelial cells and the stroma in the villous core. This structure is suitable to ensure enough maternal blood supply and proper nutrition and oxygenation and of the embryo. Moreover, thin Cy facilitates its fusion with Sy, what is known as syncytial fusion that helps syncytial growth and proliferation. This ensures that $\mathrm{Cy}$ at the tips of the villi grows out to penetrate into the endometrium for proper invasion of the spiral arteries which is a key event in normal placentation $[4,17]$.

Early in pregnancy, the placenta and embryo develop in a state of low oxygen tension to protect the developing fetus from the dangerous effects of reactive oxygen species (ROS). With the onset of maternal blood flow, the placental oxygen concentration gradually rises and is used for generation of adenosine triphosphate (ATP) by the mitochondria with production of ROS [12,21,22]. Physiological ROS levels play a vital role in placental development and functions. Under normal conditions, the placental antioxidant mechanisms could balance the effects of ROS and this redox state helps to regulate blood pressure $[11,23]$.

However, in PE, placental ischemia induces mitochondrial dysfunction that results in reduced oxygen consumption and a greater reliance on glycolytic metabolism with excessive ROS production that induces OS that is worsened by the reduced activity of anti-oxidant enzymes. Placental ischemia with OS may aggravate the placental dysfunction and stimulate the placental release of anti-angiogenic mediators. These mediators eventually cause endothelial dysfunction not only in the placenta but also in the kidney with vasoconstriction and hypertension [8,12,24, 25].

Thus, the current study showed structural changes in PE placentas, as thinning out of the Sy with reduced number of microvilli together with multiple syncytial knots and aggregations of glycogen. This agrees with other studies that reported the same features of immaturity and thinning $[14,21,26,27,28]$. It was concluded that reduction of the villous tree is a hallmark of PE due to the sever hypoxia and ROS. This may lead to shallow trophoblastic invasion and improper implantation which is a major characteristic of PE placentas [14,28,29]. Also, syncytial knots ; which were mentioned as a sign of immaturity; were reported in 10-15\% of the terminal villi in normal placentas, but were more pronounced in cases of low birth weight and low placental weight, as in PE [1,30]. They were attributed to hypoxia with the resulting malnutrition and immaturity of the villi as well as hormonal disturbances [1]. Moreover, these knots exhibit heavily condensed chromatin which is considered a feature of Sy apoptosis that might be induced by the excessive hypoxia together with high lysosomal activity in the trophoblastic cells. Hypoxia also causes dilatation and stress of the ER which is considered a sign of necrosis [14,31]. Also, cytochrome C; a mitochondrial discharge; was said to increase caspase activation with trophoblastic apoptosis [12,21].

In PE, OS causes structural and physiological damage to DNA, RNA and lipids with a significant cellular damage and death. Thus, it could be ruled out that hypoxia of the trophoblast cells, together with reduction of ATP biosynthesis and OS may induce apoptosis of placental cells [12,32,33]. In the same context, accumulation of glycogen in the trophoblasts of PE placentas may be considered a metabolic marker of immaturity, as it was stated that glycogen content tends to decrease as cells reach maturity [14].

Vacuoles and edematous spaces detected in the trophoblasts and villous core in PE placentas in the present study have been mentioned also by some authors who concluded that these vaculations might be considered a sign of apoptosis $[14,21,26,27]$. Slight vacuolation may be present in the normal placenta and is hypothesized to be a physiological form of cell degradation which promotes placental survival and maturation [17]. On the other hand, PE and its accompanying hypoxia may enhance the lysosome/vacuole functions resulting in a widespread cytoplasmic vacuolation that definitely would increase the thickness of the placental barrier and significantly compromise the utero-placental blood flow. Moreover, thickening of the subtrophoblastic membrane and walls of the villous capillaries might significantly affect feto-maternal exchange across the placenta and reduce embryonic oxygenation and nutrition [4].

Also, the thickness of the placental barrier might be increased by the deposition of fibrin in the villous core as was demonstrated in the current study and mentioned by many authors $[1,21,28,33]$. Fibrin deposition has been attributed to an increased placental antifibrinolytic potential. In PE, there is an imbalance between the plasminogen activators ;important factors in fibrinolysis; and their inhibitors with elevated levels of the inhibitors and the consequent deposition of fibrin [14,33,34]. Again, this would increase the thickness of the placental barrier and negatively affect the utero-placental blood flow that might add to the hypoxic state with its consequent trophoblastic necrosis [28,35]. 
The fibrinoid degeneration observed in PE placentas in the current study has been mentioned by some authors [1,14]. An explanation was given, that the fibrinoid degeneration is a special form of necrosis that may result from deposition of fibrin that leaked outside the vessels together with other molecules derived from blood clotting or the hypoxiainduced degenerative processes. This fibrinoid material replaces the disrupted SY on the terminal villi acting as a kind of barrier adding to the reduced materno-fetal exchange [4].

\section{Conclusion}

The underlying vasculopathies of the uterine spiral arteries with abnormal trophoblastic invasion might result in hypoxia and OS which are considered important players in PE. Thus, this might be presented as an explanation for the placental structural abnormalities such as trophoblastic necrosis, reduction of the villous tree and increased thickness of the placental barrier with the resulting impaired utero-placental flow and materno-fetal exchange and their deleterious effects on both the placenta and fetus [1,14].

\section{Compliance with ethical standards}

\section{Acknowledgments}

The authors thank the staff of the Department of Gynecology and Obstetrics, Faculty of Medicine, Alexandria University for their help for obtaining the samples of the placentas and their help in follow up of the cases subjected to the study.

\section{Disclosure of conflict of interest}

The authors have declared that no competing interest exists.

\section{Statement of ethical approval}

All the steps of the study were approved by the Committee of Ethics in the Faculty of Medicine, Alexandria University.

\section{Statement of informed consent}

Informed consent was obtained from all individual participants included in the study.

\section{References}

[1] Salmani D, Purushothaman S, Somashekara SC, Gnanagurudasan E, Sumangaladevi K, Harikishan R, Venkateshwarareddy M. Study of structural changes in placenta in pregnancy-induced hypertension. Journal of Natural Science, Biology and Medicine. 2014; 5(2): 352-355.

[2] Burton GJ, Fowden AL. The placenta: a multifaceted, transient organ. Philosophical transactions of the Royal Society of London. Series B. Biological Sciences. 2015; 370(1663): 20140066.

[3] Singh V. Extraembryonic membranes and twinning. In: Textbook of Clinical Embryology. 1st Edition Elsevier India. 2012; 57-75.

[4] Abdelghany AH, Eissa TM, Idris S. Study of the ultrastructure of the placenta in gestational Diabetes mellitus. International Journal of Anatomical Variations. 2018; 11(1): 4-10.

[5] Whitley GS, Cartwright JE. Cellular and molecular regulation of spiral artery remodeling: lessons from the cardiovascular field. Placenta. 2010; 31: 465-474.

[6] Rana S, Lemoine E, Granger JP, Karumanchi A. Preeclampsia: Pathophysiology, Challenges, and Perspectives. Circulation Research. 2019; 124: 1094-1112.

[7] Chaiworapongsa T, Chaemsaithong P, Yeo L, Romero R. Pre-eclampsia part 1: current understanding of its pathophysiology. Nature Reviews Nephrology. 2014; 10(8): 466-480.

[8] Vangrieken P, Al-Nasiry S, Bast A, Leermakers PA, Tulen CBM, Schiffers PMH, van Schooten FJ, Remels AHV. Placental Mitochondrial Abnormalities in Preeclampsia. Reproductive Sciences. 2020.

[9] Amaral LM, Wallace K, Owens M and LaMarca B. Pathophysiology and Current Clinical Management of Preeclampsia. Current Hypertension Report. 2017; 19(8): 61. 
[10] Aouache R, Biquard L, Vaiman D, Miralles F. Oxidative Stress in Preeclampsia and Placental Diseases. International Journal of Molecular Sciences. 2018; 19(5): 1496.

[11] Jena MK, Sharma NR, Petitt M, Maulik D, Nayak NR. Pathogenesis of Preeclampsia and Therapeutic Approaches Targeting the Placenta. Biomolecules. 2020; 10(6): 593.

[12] Vaka VR, McMaster KM, Cunningham MW, Ibrahim T, Hazlewood R, Usry N, Cornelius D, Amaral LM, and LaMarca B. The role of mitochondrial dysfunction and ROS in mediating hypertension in the RUPP rat model of preeclampsia. Hypertension. 2018; 72(3): 703-11.

[13] World Health Organization, 2011 WHO Recommendations for prevention and treatment of pre-eclampsia and eclampsia.

[14] Roland CR, Hu J, Ren CE, Chen5 H, Li J, Varvoutis MS, Leaphart LW, Byck DB, Zhu X, Jiang SW. Morphological changes of placental syncytium and their implications for the pathogenesis of preeclampsia. Cellular and Molecular Life Sciences. 2016; 73(2): 365-376.

[15] Bancroft J, Stevens A. Theory and Practice of Histological Techniques. 2nd Ed. New York: Churchill-Livingston. 1982; 131-135.

[16] Hayat MA. Principles and techniques of electron microscopy: biological applications. 3rd ed. New York: CRC Press. 1989; 24-74.

[17] Hassan A, Essa TM. Ultrastructure of the placenta in gestational diabetes mellitus. Anatomy International Journal of Experimental and Clinical Anatomy. 2016; 10(3): 160-69.

[18] Palaskar A, Choudhary KR, Mayadeo NM. Foeto placental weight relationship in normal pregnancy, pre-eclampsia and eclampsia. Bombay Hospital Jaipur. 2001; 43: 361-3.

[19] Majumdar S, Dasguptha H, Bhattacharya K, Bhattacharya A. A study of placenta in normal and hypertensive pregnancies. Journal of the Anatomical Society of India. 2005; 54: 34-8.

[20] Gunnarsdottir J, Cnattingius S, Lundgren M, Selling K, Högberg U, Wikström AK. Prenatal exposure to preeclampsia is associated with accelerated height gain in early childhood. Plos One. 2018; 13(2): e0192514.

[21] Selim ME, Elshmry NG, Rashed EA. Electron and scanning microscopic observations on the syncytiotrophoblast microvillous membrane contribution to preeclampsia in early placental rats. J Blood Disorders Transf. 2013; 4: $1-8$.

[22] Tang X., Luo Y.X., Chen H.Z., Liu D.P. Mitochondria, endothelial cell function, and vascular diseases. Frontiers in Physiology. 2014; 5: 175.

[23] Nicolson GL. Mitochondrial Dysfunction and Chronic Disease: Treatment With Natural Supplements. Integrative Medicine (Encinitas). 2014; 13(4): 350-43.

[24] Kintiraki E, Papakatsika S, Kotronis G, Goulis DG, Kotsis V. Pregnancy-Induced hypertension. Hormones. 2015; 14(2); 211-23.

[25] Z96 Choi S, Kim JA, Li HY, Lee SJ, Seok YS, Kim TH, Han KH, Park MH, Cho GJ, Suh SH. Altered Redox State Modulates Endothelial KCa2.3 and KCa3.1 Levels in Normal Pregnancy and Preeclampsia. Antioxidants Redox Signal. 2019; 30: 505-519.

[26] Ishihara N, Matsuo H, Murakoshi H, Laoag-Fernandez JB, Samoto T, Mauruo T. Increased apoptosis in the syncytiotrophoblast in human term placentas complicated by either preeclampsia or intrauterine growth retardation. American Journal of Obstetrics \& Gyncecology. 2002; 186(1): 158-166.

[27] Huang Q, Li J, Wang F, Oliver MT, Tipton T, Gao Y, Jiang SW. Syncytin-1 modulates placental trophoblast cell proliferation by promoting G1/S transition. Cell Signal. 2013; 25(4): 1027-35.

[28] Sankar KD, Bhanu PS, Kiran S, Ramakrishna BA, Shanthi V. Vasculosyncytial membrane in relation to syncytial knots complicates the placenta in preeclampsia: a histomorphometrical study. Anatomy and Cell Biology. 2012; 45(2): 86-91.

[29] Nahar L, Nahar K, Hossain MI, Yasmin H, Annur BM. Placental changes in pregnancy induced hypertension and its impacts on fetal outcome. Mymensingh Medical Journal. 2015; 24(1): 9-17.

[30] Roland CS, Hu J, Ren CE, Chen H, Li J, Varvoutis MS, Leaphart LW, Byck DB, Zhu X, Jiang SW. Morphological changes of placental syncytium and their implications for the pathogenesis of preeclampsia. Cellular and Molecular Life Sciences. 2016; 73(2): 365-76. 
[31] Huang Q, Chen H, Wang F, Brost BC, Li J, Gao Y, Li Z, Gao Y, Jiang SW. Reduced syncytin-1 expression in choriocarcinoma BeWo cells activates the calpain1-AIF-mediated apoptosis, implication for preeclampsia. Cellular and Molecular Life Sciences. 2014; 71(16): 3151-3165.

[32] Aouache R, Biquard L, Vaiman D, Miralles F. Oxidative Stress in Preeclampsia and Placental Diseases. International Journal of Molecular sciences. 2018; 19(5): 1496.

[33] Bobinski R, Pielesz A, Waksmańska W, Sarna E, Ulman-Włodarz I, Kania J, Mikulska M, Turbiarz A. Surface structure changes of pathological placenta tissue observed using scanning electron microscopy - a pilot study. Acta Biochemica Polonica. 2017; 64(3): 533-535.

[34] Kanfer A, Bruch JF, Nguyen G, He CJ, Delarue F, Flahault A, Nessmann C, Uzan S. Increased placental antifibrinolytic potential and fibrin deposits in pregnancy-induced hypertension and preeclampsia. Laboratory Investigation. 1996; 74(1): 253-258.

[35] Guller S, Yula YM, Han-Hsuan F, Krikun G, Abrahams VM, Mor G. The placental syncytium and the pathophysiology of preeclampsia and intrauterine growth restriction: a novel assay to assess syncytial protein expression. Annal of the New York Academy of Sciences. 2008; 1127: 129-133. 